\title{
Two-loop corrections to Bhabha scattering
}

\author{
Alexander Penin ${ }^{*}$ \\ Institut für Theoretische Teilchenphysik, Universität Karlsruhe, 76128 Karlsruhe, Germany \\ E-mail: ap@particle.uni-karlsruhe.de
}

The two-loop radiative photonic corrections to Bhabha scattering are computed in the leading order of the small electron mass expansion up to the nonlogarithmic term. After including the soft photon bremsstrahlung we obtain the infrared-finite result for the differential cross section, which can directly be applied to a precise luminosity determination of the present and future $e^{+} e^{-}$ colliders.

International Europhysics Conference on High Energy Physics

July 21st - 27th 2005

Lisboa, Portugal

\footnotetext{
* Speaker.

$\dagger$ Institute for Nuclear Research of Russian Academy of Sciences, 119899 Moscow, Russia

$\ddagger$ The work is supported in part by BMBF Grant No. 05HT4VKA/3 and Sonderforschungsbereich Transregio 9.
} 
Electron-positron Bhabha scattering plays a special role in particle phenomenology. It is crucial for extracting physics from experiments at electron-positron colliders since it provides a very efficient tool for luminosity determination. The small angle Bhabha scattering has been particularly effective as a luminosity monitor in the LEP and SLC energy range because its cross section is large and QED dominated [1]. At a future International Linear Collider the luminosity spectrum is not monochromatic due to beam-beam effects. Therefore measuring the cross section of the small angle Bhabha scattering alone is not sufficient, and the angular distribution of the large angle Bhabha scattering has been suggested for disentangling the luminosity spectrum [2]. The large angle Bhabha scattering is important also at colliders operating at a center of mass energy $\sqrt{s}$ of a few $\mathrm{GeV}$, such as such as BABAR/PEP-II, BELLE/KEKB, BES/BEPC, KLOE/DA $\Phi N E$, and VEPP$2 \mathrm{M}$, where it is used to measure the integrated luminosity [3]. Since the accuracy of the theoretical evaluation of the Bhabha cross section directly affects the luminosity determination, remarkable efforts have been devoted to the study of the radiative corrections to this process (see [1] for an extensive list of references). Pure QED contributions are particularly important because they dominate the radiative corrections to the large angle scattering at intermediate energies $1-10 \mathrm{GeV}$ and to the small angle scattering also at higher energies. The calculation of the QED radiative corrections to the Bhabha cross section is among the classical problems of perturbative quantum field theory with a long history. The first order corrections are well known (see [4] and references therein). To match the impressive experimental accuracy the complete second order QED effects have to be included on the theoretical side. The evaluation of the two-loop virtual corrections constitutes the main problem of the second order analysis. The complete two-loop virtual corrections to the scattering amplitudes in the massless electron approximation have been computed in Ref. [5], where dimensional regularization has been used for infrared divergences. However, this approximation is not sufficient since one has to keep a nonvanishing electron mass to make the result compatible with available Monte Carlo event generators [1]. Recently an important class of the two-loop corrections, which include at least one closed fermion loop, has been obtained for a finite electron mass [6] including the soft photon bremsstrahlung [7]. A similar evaluation of the purely photonic two-loop corrections is a challenging problem at the limit of present computational techniques. On the other hand in the energy range under consideration only the leading contribution in the small ratio $m_{e}^{2} / s$ is of phenomenological relevance and should be retained in the theoretical estimates. For arbitrary scattering angle even in this approximation only the two-loop corrections enhanced by a power of the large logarithm $\ln \left(m_{e}^{2} / s\right)$ have been known [8,9]. In the limit of the small scattering angle, however, the structure of the corrections is much simpler [10] that allowed for the evaluation of the corrections up to the nonlogarithmic term [11, 12]. The result for the nonlogarithmic contribution for arbitrary scattering angle has been recently obtained in Ref. [13] by employing the general theory of infrared singularities in QED, which allows to reduce the calculation in the small electron mass approximation to the analysis of a strictly massless scattering amplitude and the massive vector form factor. Below we outline the method and the result of the calculation.

The leading asymptotics of the virtual corrections cannot be obtained simply by putting $m_{e}=0$ because the electron mass regulates the collinear divergences. In addition the virtual corrections are a subject of soft divergences, which can be regulated by giving the photon a small auxiliary mass $\lambda$. The soft divergences are canceled out in the inclusive cross section when one adds the contribution of the soft photon bremsstrahlung. Thus we have to compute the two-loop virtual cor- 
rections to the four-fermion amplitude $\mathscr{A}^{(2)}\left(m_{e}, \lambda\right)$. To get the leading term in $m_{e}^{2} / s$ we develop the method applied first in Ref. [14] to the analysis of the two-loop corrections to the fermion form factor in an Abelian gauge model with mass gap. The main idea is to construct an auxiliary amplitude $\tilde{\mathscr{A}}^{(2)}\left(m_{e}, \lambda\right)$, which has the same structure of the infrared singularities but is simpler to evaluate. Then the difference $\mathscr{A}^{(2)}-\tilde{\mathscr{A}}^{(2)}$ has a finite limit $\delta \mathscr{A}^{(2)}$ as $m_{e}, \lambda \rightarrow 0$. This quantity does not depend on the regularization scheme for $\mathscr{A}^{(2)}$ and $\tilde{\mathscr{A}}^{(2)}$ and can be evaluated in dimensional regularization in the limit of four space-time dimensions. In this way we obtain $\mathscr{A}^{(2)}\left(m_{e}, \lambda\right)=\tilde{\mathscr{A}}^{(2)}\left(m_{e}, \lambda\right)+\delta \mathscr{A}^{(2)}+\mathscr{O}\left(m_{e}, \lambda\right)$. The singular dependence of the virtual corrections on infrared regulators obeys evolution equations, which imply factorization of the infrared singularities [15]. One can use this property to construct the auxiliary amplitude $\tilde{\mathscr{A}}^{(2)}\left(m_{e}, \lambda\right)$. For example, the collinear divergences are known to factorize into the external line corrections [16]. This means that the singular dependence of the corrections to the four-fermion amplitude on $m_{e}$ is the same as of the corrections to (the square of) the electromagnetic fermion form factor. The remaining singular dependence of the amplitude on $\lambda$ satisfies a linear differential equation [15] and the corresponding soft divergences exponentiate. A careful analysis shows that for pure photonic corrections $\tilde{\mathscr{A}}^{(2)}\left(m_{e}, \lambda\right)$ can be constructed of the two-loop corrections to the form factor and the products of the one-loop contributions. We have checked that in dimensional regularization the structure of the infrared divergences of the auxiliary amplitude obtained in this way agrees with the one given in Refs. $[5,17]$. Thus in our method the infrared divergences, which induce the asymptotic dependence of the virtual corrections on the electron and photon masses, are absorbed into the auxiliary factorized amplitude while the technically most nontrivial calculation of the matching term $\delta \mathscr{A}^{(2)}$ is performed in the massless approximation. Note that the method does not require a diagram-by-diagram subtraction of the infrared divergences since only a general information on the infrared structure of the total two-loop correction is necessary to construct $\tilde{\mathscr{A}}^{(2)}\left(m_{e}, \lambda\right)$. For the calculation of the matching term $\delta \mathscr{A}^{(2)}$ beside the one-loop result one needs the two-loop massless corrections [5, 18]. For the calculation of $\tilde{\mathscr{A}}^{(2)}\left(m_{e}, \lambda\right)$ one needs also the two-loop correction to the form factor for $\lambda \ll m_{e} \ll s$ which can be found in Ref. [19]. Let us now present our result. We define the perturbative expansion for the Bhabha cross section in the fine structure constant $\alpha$ as follows: $\sigma=\sum_{n=0}^{\infty}\left(\frac{\alpha}{\pi}\right)^{n} \sigma^{(n)}$. The leading order differential cross section reads

$$
\frac{\mathrm{d} \sigma^{(0)}}{\mathrm{d} \Omega}=\frac{\alpha^{2}}{s}\left(\frac{1-x+x^{2}}{x}\right)^{2},
$$

where $x=(1-\cos \theta) / 2$ and $\theta$ is the scattering angle. Virtual corrections taken separately are infrared divergent. To get a finite scheme independent result we include the contribution of the soft photon bremsstrahlung into the cross section. Thus the second order corrections can be represented as a sum of three terms $\sigma^{(2)}=\sigma_{v v}^{(2)}+\sigma_{s v}^{(2)}+\sigma_{s s}^{(2)}$, which correspond to the two-loop virtual correction including the interference of the one-loop corrections to the amplitude, one-loop virtual correction to the single soft photon emission, and the double soft photon emission, respectively. When the soft photon energy cut is much less than $m_{e}$, the result for the two last terms in the above equation is known in analytical form and can be found e.g. in Ref. [8]. The second order correction can be decomposed according to the asymptotic dependence on the electron mass

$$
\frac{\mathrm{d} \sigma^{(2)}}{\mathrm{d} \sigma^{(0)}}=\delta_{2}^{(2)} \ln ^{2}\left(\frac{s}{m_{e}^{2}}\right)+\delta_{1}^{(2)} \ln \left(\frac{s}{m_{e}^{2}}\right)+\delta_{0}^{(2)}+\mathscr{O}\left(m_{e}^{2} / s\right),
$$




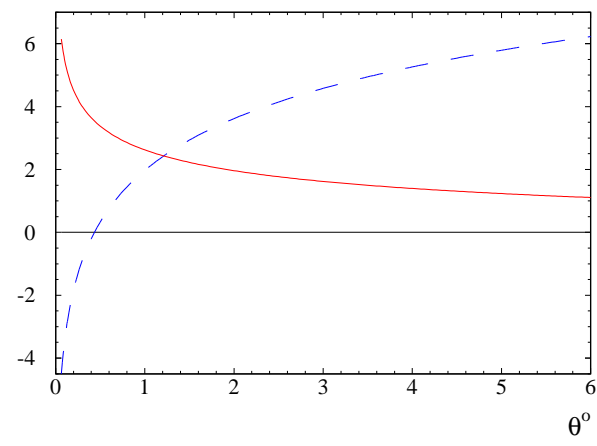

(a)

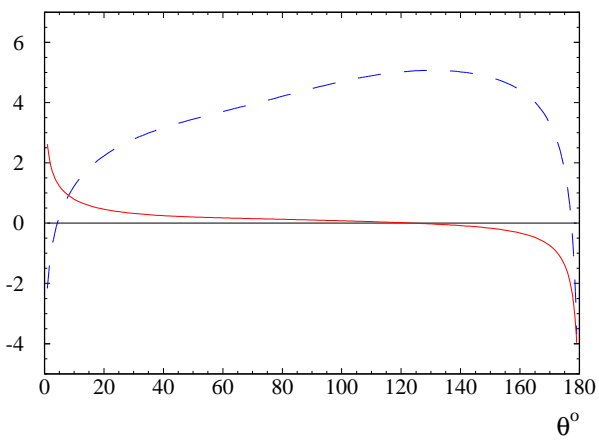

(b)

Figure 1: Logarithmically enhanced (dashed line) and nonlogarithmic (solid line) second order corrections to the differential cross section of the Bhabha scattering as functions of the scattering angle for (a) $\sqrt{s}=$ $100 \mathrm{GeV}$ and (b) $\sqrt{s}=1 \mathrm{GeV}$, in units of $10^{-3}$.

where the coefficients $\delta_{i}^{(j)}$ are independent on $m_{e}$. The result for the logarithmically enhanced corrections is known (see [8] and references therein). The analytical expression for the nonlogarithmic term is rather lengthy and can be found in [13]. Numerical value of the second order correction to the differential cross section $(\alpha / \pi)^{2} \mathrm{~d} \sigma^{(2)} / \mathrm{d} \sigma^{(0)}$ is plotted as the function of the scattering angle for the small angle Bhabha scattering at $\sqrt{s}=100 \mathrm{GeV}$ on Fig. (1a) and for the large angle Bhabha scattering at $\sqrt{s}=1 \mathrm{GeV}$ on Fig. (1b). We separate the logarithmically enhanced corrections given by the first two terms of Eq. (2) and nonlogarithmic contribution given by the last term of this equation. All the terms involving a power of the logarithm $\ln \left(\varepsilon_{c u t} / \varepsilon\right)$ are excluded from the numerical estimates because the corresponding contribution critically depends on the event selection algorithm and cannot be unambiguously estimated without imposing specific cuts on the photon bremsstrahlung.

Finally, we would like to mention that for the large angle scattering above the electroweak scale the two-loop electroweak corrections could be important. In the case of $e^{+} e^{-} \rightarrow \mu^{+} \mu^{-}$ annihilation the corrections enhanced in the high energy limit by a power of the large logarithm $\ln \left(M^{2} / s\right)$, where $M$ stands for $W$ or $Z$ boson mass, have been computed in [20]. They dominate the electroweak corrections for the energies $\sqrt{s} \gtrsim 500 \mathrm{GeV}$ characteristic to ILC. This analysis can be generalized to the large angle Bhabha scattering by adding the $t$-channel contribution.

To conclude, we have derived the two-loop radiative photonic corrections to Bhabha scattering in the leading order of the small electron mass expansion up to nonlogarithmic term. The nonlogarithmic contribution has been found numerically important for the practically interesting range of scattering angles. Together with the result of Refs. [6, 7] for the corrections with the closed fermion loop insertions our result gives a complete expression for the two-loop virtual corrections. It should be incorporated into the Monte Carlo event generators to match the demands of the present and future electron-positron colliders for the accuracy of the luminosity determination. 


\section{References}

[1] S. Jadach et al. in G. Altarelli, T. Sjöstrand and F. Zwirner (eds.), Physics at LEP2, CERN-96-01, hep-ph/9602393; G. Montagna, O. Nicrosini, and F. Piccinini, Riv. Nuovo Cim. 21N9, 1 (1998).

[2] N. Toomi, J. Fujimoto, S. Kawabata, Y. Kurihara, and T. Watanabe, Phys. Lett. B 429, 162 (1998); R.D. Heuer, D. Miller, F.Richard, and P.M. Zerwas, (eds.), TESLA Technical design report. Pt. 3: Physics at an $e^{+} e^{-}$linear collider, DESY-01-011C.

[3] C. M. Carloni Calame, C. Lunardini, G. Montagna, O. Nicrosini, and F. Piccinini, Nucl. Phys. B584, 459 (2000).

[4] M. Bohm, A. Denner, and W. Hollik, Nucl. Phys. B304, 687 (1988).

[5] Z. Bern, L. Dixon, and A. Ghinculov, Phys. Rev. D 63, 053007 (2001).

[6] R. Bonciani, A. Ferroglia, P. Mastrolia, and E. Remiddi, Nucl. Phys. B701, 121 (2004).

[7] R. Bonciani, A. Ferroglia, P. Mastrolia, E. Remiddi, and J.J. van der Bij, Nucl. Phys. B716, 280 (2005).

[8] A.B. Arbuzov, E.A. Kuraev, and B.G. Shaikhatdenov, Mod. Phys. Lett. A 13, 2305 (1998)

[9] E.W. Glover, J.B. Tausk, and J.J. van der Bij, Phys. Lett. B 516, 33 (2001).

[10] V.S. Fadin, E.A. Kuraev, L.N. Lipatov, N.P. Merenkov, and L. Trentadue, Fhys. Atom. Nucl. 56, 1537 (1993) [Yad. Fiz. 56145 (1993)].

[11] A.B. Arbuzov, V.S. Fadin, E.A. Kuraev, L.N. Lipatov, N.P. Merenkov, and L. Trentadue, Nucl. Phys. B485, 457 (1997).

[12] S. Jadach, M. Melles, B.F.L. Ward, and S.A. Yost, Phys. Lett. B 450, 262 (1999).

[13] A.A. Penin, Phys. Rev. Lett. 95, 010408 (2005); hep-ph/0508127.

[14] B. Feucht, J.H. Kühn, A.A. Penin, and V.A. Smirnov, Phys. Rev. Lett. 93, 101802 (2004).

[15] D.R. Yennie, S.C. Frautschi, and H. Suura, Ann. Phys. 13, 379 (1961). A.H. Mueller, Phys. Rev. D 20 , 2037 (1979); J.C. Collins, Phys. Rev. D 22 (1980) 1478.

[16] J. Frenkel and J.C. Taylor, Nucl. Phys. B116, 185 (1976).

[17] S. Catani, Phys. Lett. B 427, 161 (1998).

[18] G. Kramer and B. Lampe, Z. Phys. C 34, 497 (1987); 42, 504(E) (1989); T. Matsuura, S.C. van der Marck, and W.L. van Neerven, Nucl. Phys. B319, 570 (1989).

[19] G.J.H. Burgers, Phys. Lett. B 164, 167 (1985); P. Mastrolia and E. Remiddi, Nucl.Phys. B664, 341 (2003).

[20] J.H. Kühn, A.A. Penin, and V.A. Smirnov, Eur. Phys. J. C 17, 97 (2000); Nucl. Phys. Proc. Suppl. 89, 94 (2000); J.H. Kühn, S. Moch, A.A. Penin, and V.A. Smirnov, Nucl. Phys. B616, 286 (2001); B648, 455(E) (2003); B. Jantzen, J.H. Kühn, A.A. Penin, and V.A. Smirnov, Phys. Rev. D 72, 051301(R) (2005); Nucl. Phys. B731, 188 (2005). 